\title{
nature
}

cell biology

\section{Molecule pages live}

The Alliance for Cellular Signaling (AfCS) is now over two years into its ambitious ten-year plan to generate a new platform for understanding signal transduction. The project is essentially divided into two main areas: first, a multilab effort to map signalling pathways quantitatively in two defined murine cell systems; second, the generation of a relational database designed to capture all significant published knowledge on a comprehensive set of signalling molecules. We have previously described this open access community effort, and Nature Publishing Group's involvement in it, in some detail (see NCB; DOI: 10.1038/ncb1202-e273 and our supplement of 2002 at http://www.nature.com/cgitaf/DynaPage.taf?file=/nature/journal/v420/n6916/index.html\#allianc e) and we invite our readers to explore further at http://www.signaling-gateway.org/.

In December the project reached another milestone with the release of the first handful of authored Molecule Pages (MPs; http://www.signaling-gateway.org/molecule/). Although 3,500 MPs, each on an individual signalling protein, have populated the database for some time, these entries are based on automated data-feeds that are updated monthly, collating essential molecule-specific information from a complementary set of existing databases (including names/synonyms, sequence-, domain- and motif-information, structure data and orthologues/paralogues). The new-generation full MPs add a further important layer to this useful resource - expert authors manually enter literature-derived information about their favourite molecule. The basic underlying philosophy is to distil the published literature to capture only facts that have stood the test of time for reproducibility, and importantly, to enter this information in the relevant context of the experimental conditions and system, as well as to integrate molecular attributes into their proper regulatory context. This highly structured quantitative information on molecular function, intermolecular relationships and sub-cellular localization is each directly associated with references to the relevant literature. MPs are designed ultimately to allow both systems biology and comprehensive molecule-specific interrogation, setting the database apart from most others.

To ensure that the MPs are authoritative, they are authored by established specialists and subjected to rigorous independent peer review by Nature. As such, the database is unique in its ambitious level of curation. MPs are fully citable and should be regarded as a bona fide publication with the important benefit of being updated annually. Authoring MPs, and indeed peer reviewing them, is a laudable and by no means insignificant feat, not least because there is a commitment attached to the regular updating of each entry to ensure timely representation of the often rapidly expanding knowledge-base. The database thus relies heavily on the goodwill of its contributors. We very much hope that you will play an active part, be it as author, referee, or simply by sending comments. This is a project for the community by the community and it will only flourish with its active participation. As such, we also suggest that proper academic credit be given to those putting in the time.

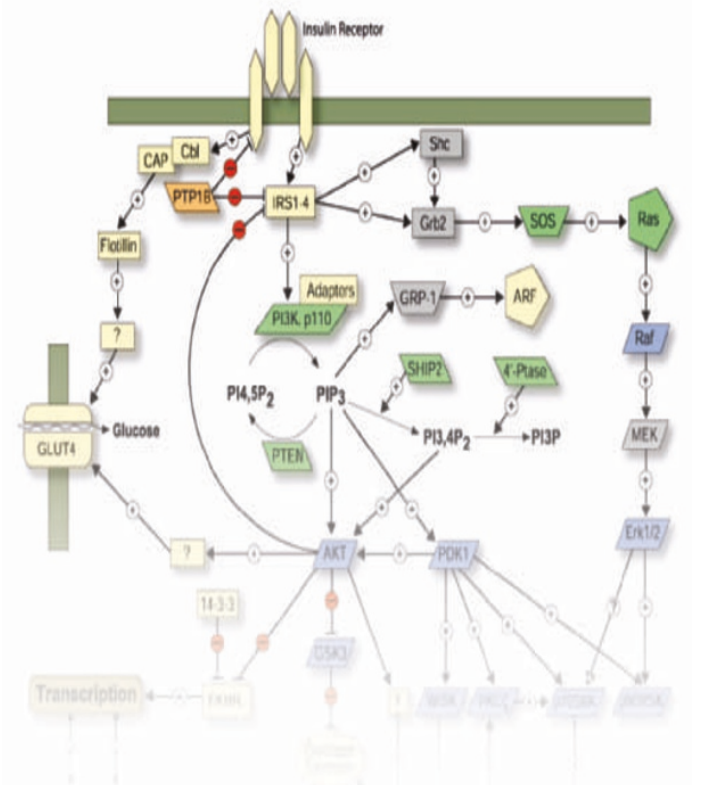

\section{Value added}

The beginning of the year is a good place to revisit some of the possibly less noticeable places beyond the selection and peer review process (NCB; DOI:10.1038/ncb0703-583) where we add value to your paper. Significant editorial energies are invested into developmental editing, exhaustive sub-editing and signposting of articles with News and Views, and global press releases. In addition, our dedicated production team ensures that $\mathrm{NCB}$, both in print and online, benefits from the highest journal production standards. Our art editor recasts not only all Review and News and Views figures, but also relabels the figures of primary research articles to maximize clarity and to produce an appearance that is easy on the eye of the browsing reader. We publish final versions of every paper online significantly before the print issue, and this constitutes the official publication date. Importantly, we regard Supplementary Information as an integral part of a paper, and the pdf versions seamlessly integrate Supplementary Information into the main body of the paper (in as far as the data allows). Some other notable features of NCB include the pre-submission inquiry option, increasingly efficient cross-journal referrals, referee feedback letters, as well as subject-based focus issues and supplements. In the future, we aim to provide paper-specific citation, and statistics for paper access and media coverage. We are developing active linking of text to online resources (encyclopaedic and databases), and we are exploring the display of online data in a dynamic vector graphics format. Naturally, we would very much welcome feedback and ideas of what you would like to see NCB do. Meanwhile, we hope the papers that appear in the journal bear the testimony of this involved process in being at once pre-eminent, accessible and authoritative. 